\title{
Overexpression of the Glyoxalase II Gene Leads to Enhanced Salinity Tolerance in Brassica Juncea
}

\author{
Mukesh Saxena ${ }^{1}$, Suchandra Deb Roy ${ }^{1}$, Sneh-Lata Singla-Pareek ${ }^{2}$, Sudhir Kumar Sopory ${ }^{2}$ \\ and Neera Bhalla-Sarin ${ }^{1, *}$ \\ ${ }^{1}$ School of Life Sciences, Jawaharlal Nehru University, New Delhi-110067, India \\ ${ }^{2}$ Plant Molecular Biology, I.C.G.E.B., Aruna Asaf Ali Marg, New Delhi-110067, India
}

\begin{abstract}
Engineering of salinity tolerance in agronomically important crop plants is required to increase their productivity by enabling them to grow in saline soils, which are otherwise left uncultivated. Since an increase in the enzymes of glyoxalase system has been shown to impart salinity tolerance in the model plant tobacco, we used the glyoxalase II gene for engineering salinity tolerance in an important oil yielding crop, Brassica juncea. The transgenic plants of $B$. juncea overexpressing the glyoxalase II gene showed higher salinity tolerance as compared to the untransformed control plants as observed by delayed senescence in leaf discs at $400 \mathrm{mM}$ and $800 \mathrm{mM} \mathrm{NaCl}$ in T1 generation. The percentage of germination of the T2 transgenic seeds was higher at $150 \mathrm{mM}$ and $200 \mathrm{mM} \mathrm{NaCl}$ as compared to the seeds of untransformed plants. This for the first time demonstrates the applicability of utilizing the glyoxalase II gene for enhanced salinity tolerance in an oilseed crop plant B. juncea.
\end{abstract}

Keywords: Brassica juncea, glyoxalase II, salinity tolerance, methylglyoxal, $\mathrm{NaCl}$.

\section{INTRODUCTION}

As the population of the world is increasing, new means of improving crop productivity must be found to increase the resources available. In contrast to monogenic traits for engineered resistance to the pests and herbicides, the genetically more complex responses to abiotic stresses are difficult to control and engineer. In the recent years, because of an increase in the level of salt in water and soil, salinity has become a major threat to agricultural productivity worldwide. Present engineering strategies for salinity tolerance rely on the transfer of one or more genes that are either involved in the signaling pathways or that encode enzymes required for the functional and structural protectants, such as osmolytes and antioxidants or that encode the proteins that confer stress tolerance [1-3]. However, considering the complex metabolic response of the plants under stress, there is still need to show that the other candidate genes also function equally well in conferring salinity tolerance.

The glyoxalase system consists of two enzymesglyoxalase I (EC 4.4.1.5, lactoylglutathione lyase) and glyoxalase II (EC 3.1.2.6, hydroxacylglutathione hydrolase). These enzymes act coordinately to convert cyotoxic methylglyoxal and other 2-oxoaldehydes to their 2-hydroxyacids using glutathione as a cofactor [4]. Methylglyoxal is a potent cytotoxic compound and its cytotoxicity is mediated by the inhibition of protein synthesis and interaction with nucleic acids. It has been shown to inhibit the activity of

*Address correspondence to this author at the School of Life Sciences, Jawaharlal Nehru University, New Delhi-110067, India; Tel: 91-11-6704523 Fax: 91-11-26742580; E-mail: neerasarin@ rediffmail.com various enzymes of glycolytic pathway under in vitro conditions [5-8]. It has also been shown to arrest growth in G1 phase of the cell cycle through inhibition of DNA synthesis $[9,10]$. However, glutathione is a well known important antioxidant involved in the detoxification of reactive oxygen species.

In higher plants, glyoxalase I activity was found to be correlated with the mitotic index [11]. An inverse correlation between glyoxalase I activity and differentiation in vitro was also observed in plants $[12,13]$. Glyoxalase I from tomato and Brassica juncea has been shown to be upregulated under different abiotic stresses [14, 15]. Moreover; the overexpression of glyoxalase I from $B$. juncea has been shown to impart tolerance in tobacco plants under salinity and heavy metal stress [15].

Unlike glyoxalase I, glyoxalase II is a less studied component of glyoxalase system in plants. The expression of the glyoxalase II gene has only been studied in Arabidopsis [16], B. juncea [17], and rice [18]. Reports in rice and $B$. juncea showed an upregulation of the transcript under stress condition. Interestingly, the overexpression of glyoxalase II in glyoxalase $I$ overexpressing lines of tobacco showed improved tolerance as compared to the plants overexpressing glyoxalase I or gyoxalase II [19]. We transferred glyoxalase II cDNA of rice in the important oil yielding crop, Brassica juncea. The transgenic plants overexpressing glyoxalase II showed significant tolerance to high concentration of $\mathrm{NaCl}$, suggesting that the glyoxalase II gene plays a vital role in salt stress alleviation. This is the first report of salt stress alleviation by overexpression of the glyoxalase II gene in an oilseed crop. 


\section{MATERIALS AND METHODS}

\section{Reagents and Supplies}

The enzymes and chemicals used for DNA manipulation were purchased from NEB, England. The oligonucleotides were obtained from Microsynth, Switzerland. Random probe labeling kit was obtained from Amersham, USA. Plant hormones, antibiotics, methylglyoxal, MOPS, etc were purchased from Sigma Chemicals,St. Louis, USA, and rest of the chemicals were from Qualigens, India.

\section{Plant Transformation and PCR Analysis}

The GV3101 strain of Agrobacterium tumefaciens was transformed with the construct $p C A M$-glyII [19] containing the glyoxalase II cDNA from rice (Os-glyII) under the control of a $35 \mathrm{~S}$ CaMV promoter (Fig. 1a). These cells carrying the construct were subsequently used to transform Brassica juncea cv Varuna according to Pental et al. [20]. Transformed shootlets regenerated on $\mathrm{MSB}_{1} \mathrm{~N}_{1}$ medium (MS medium with 6-benzyl-aminopurine and $\alpha$-naphthaleneacetic acid each at a concentration of $\left.1 \mathrm{mgl}^{-1}\right)$ containing hygromycin $\left(20 \mathrm{mg} \mathrm{l}^{-1}\right)$ and rooted on $\mathrm{MSI}_{2}$ medium (MS medium with Indole-butyric acid at a concentration of $\left.2 \mathrm{mgl}^{-1}\right)$. Primary transformants were screened by PCR analysis using glyII specific primers (5'ATGCGGATGCTGTCCAAGGCG-3' and 5'-TAAAGTTATCCTTCGCTCG-3'). These PCR positive plants were hardened and grown to maturity. They flowered and set seeds. The T1 generation were collected and germinated on MSO medium. The seedlings were rooted in selection medium ( $\mathrm{MSI}_{2}$ medium containing hygromycin at the concentration of $20 \mathrm{mgl}^{-1}$ ). Genomic DNA was isolated from the fully-grown plants [21] and used for Southern blot analysis.

\section{Southern Blot Analysis}

Southern blot analysis was done according to a modified protocol of Sambrook et al. [22]. Genomic DNA (20 $\mu \mathrm{g})$ was digested with $N c o I$ and Pst I for insert release and copy number analysis respectively, fractionated on $1 \%$ agarose gel and transferred onto a nylon membrane (MDI, India). The $\sim 1$ $\mathrm{kb}$ insert of Os glyII was used as probe. The hybridized signals were visualized using Phosphoimager (FLA-500, Japan).

\section{Northern Blot Analysis and Enzyme Assays}

Total RNA was isolated [23] and $30 \mu \mathrm{g}$ of total RNA was fractionated in a $1.5 \%$ agarose-formaldehyde gel, transferred on nylon membrane (MDI, India) by capillary flow method and UV cross-linked. The blot was stained with methylene blue to check for equal loading. Northern blots were hybridized with $\left[\alpha^{32} \mathrm{P}\right]$ dCTP labeled (random primed), purified glyII cDNA probes. The signals were detected by Phosphorimager.

For enzyme assays, protein extract was prepared by homogenizing leaf tissue in liquid nitrogen and then resuspending the powder in 1 vol (wt/vol) of extraction buffer $(0.1 \mathrm{M}$ sodium phosphate buffer $\{\mathrm{pH} 7.0\}, 50 \%$ glycerol, $16 \mathrm{mM} \mathrm{MgSO}$, $0.2 \mathrm{mM}$ PMSF and 0.2\%PVPP). The glyoxalase II enzyme activity was determined as described [16]. Three different enzyme extractions were done per sample for all independent transgenic lines. The specific activity is expressed in units per $\mathrm{mg}^{-1}$ of protein.
Leaf Disc Assay for Tolerance Against MG and Salinity Stress

Healthy and fully expanded leaves (of same age) from wild type untransformed and transgenic plants were briefly washed in deionized water and leaf discs of $1 \mathrm{~cm}$ diameter were punched out and floated in $5 \mathrm{ml}$ solution of methylglyoxal (15 mM) for 4 days or $\mathrm{NaCl}(400,800 \mathrm{mM})$ for 8 days. The chlorophyll content was measured as described [24].The experiments were done in triplicates.

\section{Salinity Tolerance of Transgenic Seeds}

$\mathrm{T}_{2}$ seeds from the wild type untransformed and transgenic plants showing higher glyoxalase II activity were germinated on MS medium containing 100, 150, $200 \mathrm{mM} \mathrm{NaCl}$ and also on MS medium without additional $\mathrm{NaCl}$. Percentage of seeds germinated was observed after 5 days. The experiments were done in triplicates of 30 seeds each.

\section{RESULTS AND DISCUSSIONS}

Agrobacterium tumefaciens GV3101 containing pCAMgly II was used to transform hypocotyls of $B$. juncea. The T0 transgenic plants were checked by PCR using the glyII specific primers. The PCR positive plants were hardened and grown to maturity. The T1 seeds from five independent PCR positive lines were germinated on MS medium and selected on hygromycin containing medium. The integration of the glyoxalase II gene in these plants was checked by PCR and Southern blot analysis. All these plants showed the amplification of $\sim 1 \mathrm{~kb}$ band corresponding to the glyoxalase II gene. (Fig. 1b). These plants were hardened and their DNA was isolated and digested with NcoI and subjected to Southern blot analysis. The plants showed the presence of $\sim 1 \mathrm{~kb}$ insert which corresponded to the Os glyI gene (Fig. 1c). To check the copy number of the transgene, the genomic DNA was also digested with the Pst enzyme which is an unique site within the T-DNA. All the transgenic plants showed a single insertion of the T-DNA within the genome (Fig. 1d). The expression of the transgene in these plants was also checked by northern blot analysis. The fusion transcript ( $g l y I I-g f p-g u s)$ was detected in four out of five transgenic lines (Fig. 1e). In one transgenic plant, low expression of the fusion transcript was also detected. The wild type untransformed control plants did not show the presence of the fusion transcript. In both Southern and northern blotting, the blots were probed with the ${ }^{32} \mathrm{P}$ labeled glyII cDNA. All these plants were also analyzed for the glyoxalase II activity. The specific activity of glyoxalase II in T1 transgenic plants was 3-6 times higher than the activity in wild type untransformed plants and it could be correlated well with the expression level of the fusion transcript (Fig. 1f).

The sensitivity of transformed plants towards $\mathrm{NaCl}$ was checked by leaf disc assays. The leaf discs of equal diameter from fully grown mature leaves of the T1 transgenic lines with higher (line 2) and lower expression (line 4) of the glyoxalase II gene and wild type untransformed plants were floated on $\mathrm{NaCl}(400 \mathrm{mM}$ and $800 \mathrm{mM})$. Incubation of the leaf discs of these transgenic lines and wild type untransformed plants in $\mathrm{NaCl}(400 \mathrm{mM}$ and $800 \mathrm{mM})$ showed delayed senescence in the transgenic plants at both the 

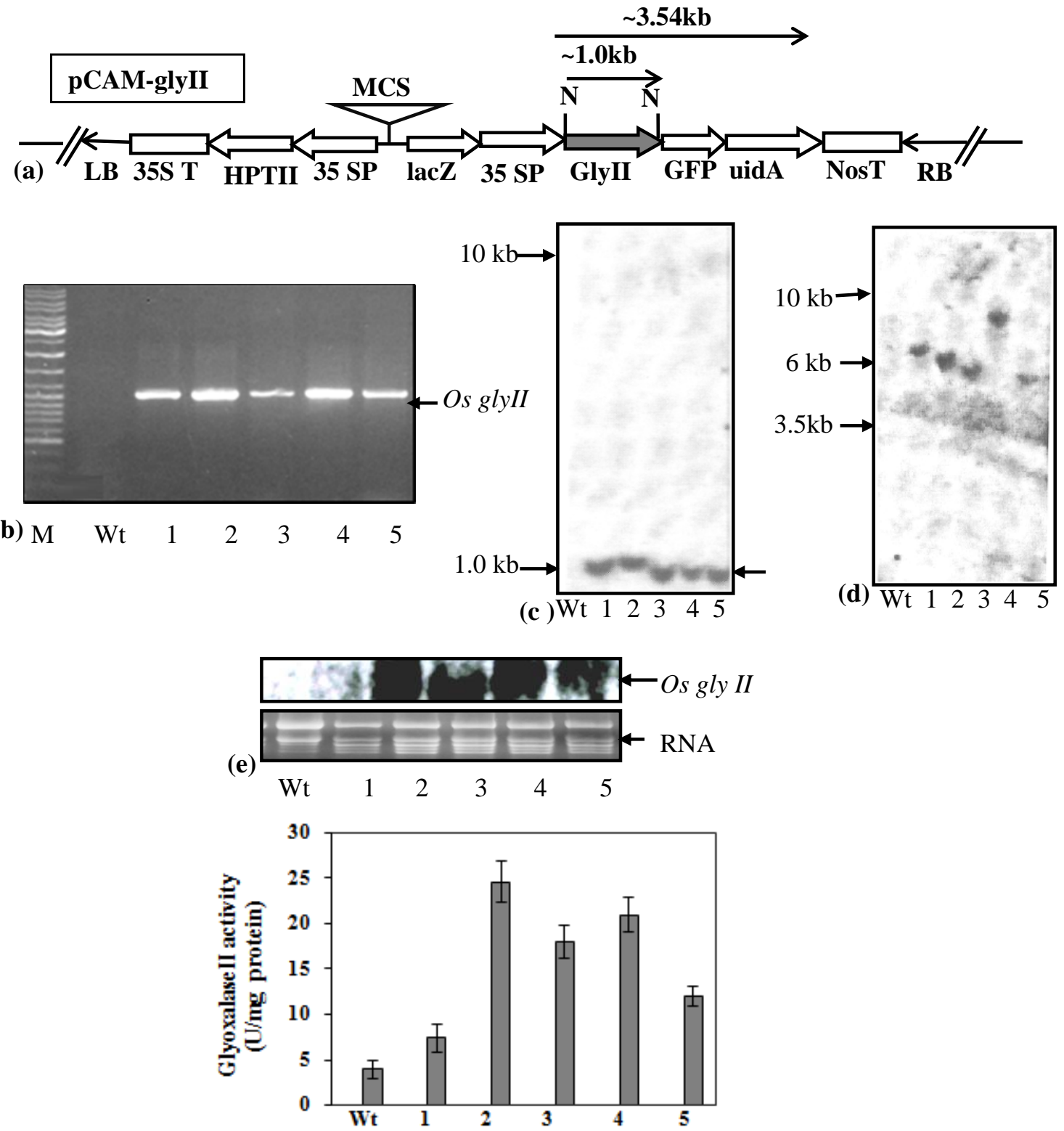

(f)

Fig. (1). (a) Schematic representation of glyoxalase II construct used for Brassica juncea transformation. (b) PCR analysis of glyoxalase II transgenics in T1 generation showing the presence of the transgene. (c) Southern blot analysis of the glyoxalase II transgenic plants vs. untransformed control plants for the presence of the transgene. (d) Southern blot analysis of the glyoxalase II transgenic plants vs. untransformed control plants for the copy number of the transgene. (e) Expression studies of the glyoxalase II transgenic plants in T1 generation by Northern analysis. (f) Specific activity of the glyoxalase II enzyme in transgenic plants vs. untransformed control plant. Total protein isolated from the transgenic as well as untransformed control plants was used for the Glyoxalase II assays in Tris buffer (pH7.2) containing $150 \mu \mathrm{M}$ SLG. Wt: Wild type untransformed plants 1-5: transgenic plants.

concentrations (Fig. 2a) as compared to wild type untransformed plant after 8 days of incubation. At both the concentrations of $\mathrm{NaCl}$ (400 and $800 \mathrm{mM})$, chlorophyll content in the leaf discs of transgenic plants was higher as compared to those from the wild type untransformed plants. Moreover, the chlorophyll content in the leaf discs from the plants with higher expression of the glyII gene (line 2) was also more than in the leaf discs of the plants having lower expression of the glyII gene (line 4) at $400 \mathrm{mM}$ and $800 \mathrm{mM}$ $\mathrm{NaCl}$ concentration. The chlorophyll content in the leaf discs of T1 transgenic plants at $400 \mathrm{mM}$ was $88 \mu \mathrm{g} / \mathrm{g}$ F.wt in line 2 and $55 \mu \mathrm{g} / \mathrm{g}$ F.wt in line 4 respectively which was $\sim 6.0$ and 3.5 fold higher as compared to the chlorophyll content in the leaf discs from untransformed control plants which was $15 \mu \mathrm{g} / \mathrm{gF} . w \mathrm{t}$. However, at $800 \mathrm{mM} \mathrm{NaCl}$, the chlorophyll content in line $2(35 \mu \mathrm{g} / \mathrm{gF} . w \mathrm{t})$ and line $4(26 \mu \mathrm{g} / \mathrm{gF} . w \mathrm{t})$ was ca. 3.0 and 4.5 fold greater as compared to the untransformed control lines in which it was $8.0 \mu \mathrm{g} / \mathrm{g} \mathrm{F.wt}$ (Fig. 2b). The leaf discs of these lines also showed enhanced tolerance against methylglyoxal. Significant difference was observed among the wild type and transgenic plants after four days of $15 \mathrm{mM}$ methylglyoxal treatment (Fig. 2c). Leaf discs from line 2 with a higher expression of the glyII gene, were greener when compared to the leaf discs of the line 4 
which had a comparatively lower expression of the transgene. The chlorophyll content in these plants further confirmed the observed delay in the senescence of the leaf discs (Fig. 2d). The chlorophyll content retained in the leaf discs of T1 transgenic plants exposed to $15 \mathrm{mM}$ MG was 12 $\mu \mathrm{g} / \mathrm{g}$ F.wt in line 4 and $22.5 \mu \mathrm{g} / \mathrm{g}$ F.wt in line 2 which was $\sim 2.5$ fold and $\sim 4.5$ fold more as compared to that in the leaf discs of wild type untransformed plants $(4.95 \mu \mathrm{g} / \mathrm{g}$ F.wt).

Salt tolerance of the glyoxalase II transgenic seeds (Line 2) was tested by their germination on MS medium containing different concentrations of $\mathrm{NaCl}$ for 5 days (Fig. 3). There was $100 \%$ germination in medium with and without $50 \mathrm{mM}$
$\mathrm{NaCl}$, in seeds from untransformed control plants as well as transgenic plants. At higher concentrations of $\mathrm{NaCl}$, a gradual decrease in the germination percentage was observed in the transgenic as well as wild type seeds. However, the percentage germination in transgenic seeds was always higher as compared to the untransformed control seeds. The germination of transgenic seeds at $100 \mathrm{mM} \mathrm{NaCl}$ was 1.4 folds while at $150 \mathrm{mM}$ it was twice as compared to the controls. At $200 \mathrm{mM} \mathrm{NaCl}$, wild type seeds showed only $3 \%$ germination as compared to $40 \%$ germination of the transgenic seeds (Table 1).

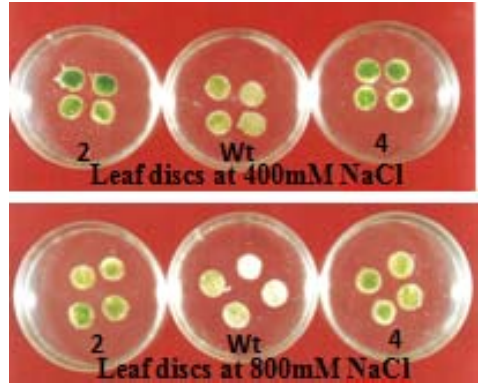

(a)

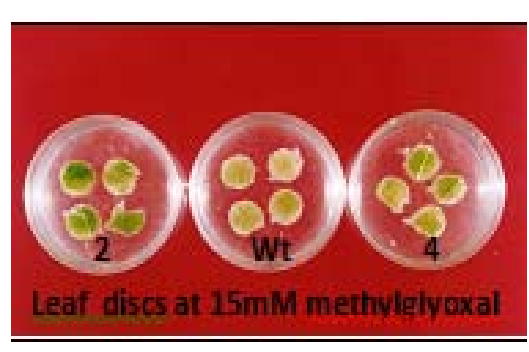

(c)

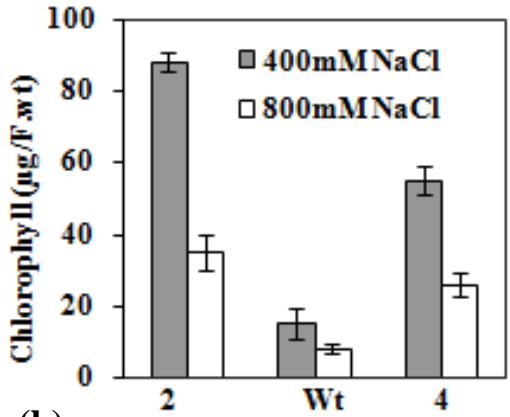

(b)

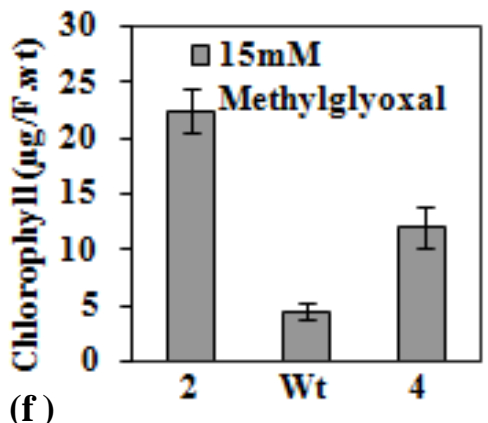

Fig. (2). Retardation of salinity and methylglyoxal induced senescence in the glyoxalase II transgenics in T1 generation. (a) Leaf discs of untransformed control plant (Wt) and transgenic plants (2 and 4) were floated on $400 \mathrm{mM}$ and $800 \mathrm{mM} \mathrm{NaCl}$. Visible differences were observed in the leaf discs of transgenic plants when compared to the untransformed control plant after 8 days. (b) Chlorophyll estimation of the leaf discs of these plants. This is in accordance with the visible effects. (c) Leaf discs of untransformed control plant and transgenic plants (2 and 4) were floated on $15 \mathrm{mM}$ methylglyoxal. Visible differences were observed in the leaf discs of transgenic plants when compared to untransformed control plant. (d) Chlorophyll estimation of the leaf discs of the above mentioned plants.

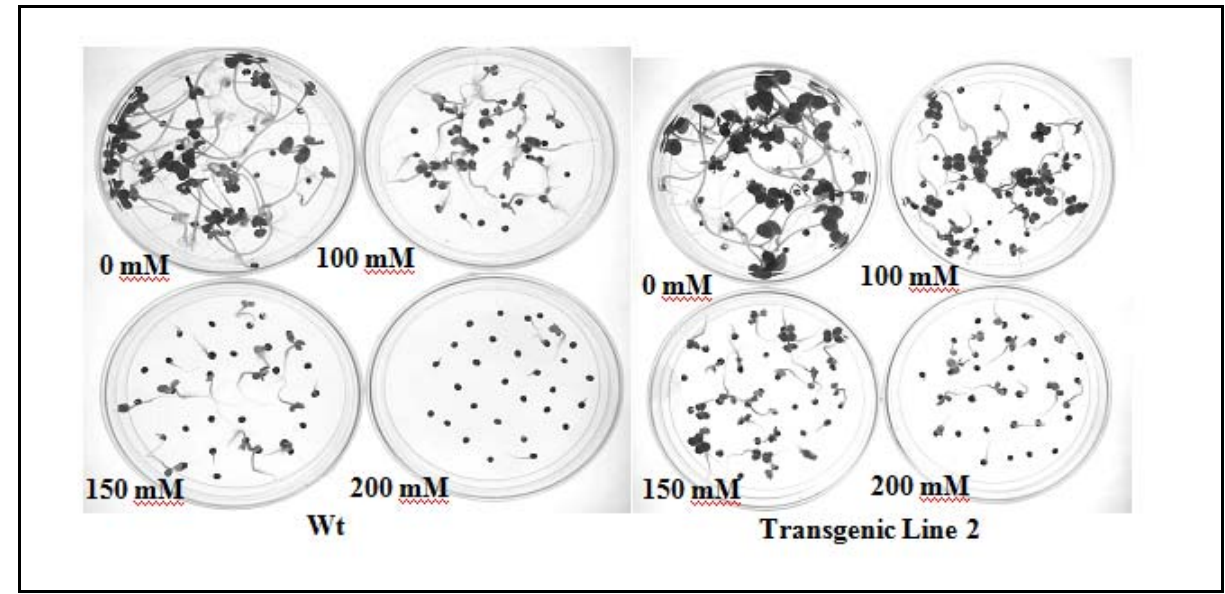

Fig. (3). Germination of T2 seeds of transgenic Brassica juncea (Line 2) vs. untransformed control plant on MS medium containing different concentrations of $\mathrm{NaCl}(0,100,150,200 \mathrm{mM})$ after 5 days. 
Table 1. Percentage Germination of Wild Type vs. Transgenic Seeds at Different Concentration of NaCl

\begin{tabular}{|c|c|c|c|c|}
\hline Concentration of NaCl & $\mathbf{0 ~} \mathbf{~ M M}$ & $\mathbf{1 0 0} \mathbf{~ m M}$ & $\mathbf{1 5 0} \mathbf{~ m M}$ & $\mathbf{2 0 0} \mathbf{~ m M}$ \\
\hline \hline $\mathrm{Wt}$ & 100 & $58.8 . \pm 3.86$ & $36.6 \pm 3.35$ & $4.44 \pm 1.92$ \\
\hline Line 2 & 100 & $82.2 \pm 1.90$ & $72.3 \pm 1.84$ & $39.96 \pm 3.35$ \\
\hline
\end{tabular}

Three different mechanisms have been suggested to be involved in stress tolerance. These include (i) ion and osmotic homeostasis, (ii) regulating cell division and growth and (iii) detoxification and cellular repair. Glyoxalase system appears to be operating through the mechanism of detoxification and cellular repair. Methylglyoxal, besides its own cytotoxicity, lowers the level of glutathione, by the formation of hemithioactal with glutathione. Under stress conditions, the increased level of methylglyoxal could further lower the glutathione level required for scavenging of reactive oxygen species, which increase under stress conditions [25]. Since the glyoxalase I has been shown to be upregulated under different abiotic stresses $[14,15]$ and an increase in the glyoxalase II transcript has been observed under various abiotic stresses in Brasssica juncea [17] and in rice [18], the overexpression of the enzymes of the glyoxalase system is presumed to be involved in stress tolerance by detoxifying methylglyoxal and elevating the level of glutathione. This has been recently demonstrated in transgenic tobacco plants overexpressing the glyoxalase I and glyoxalase II gene. These plants were able to maintain higher level of reduced glutathione and could tolerate the increased level of methylglyoxal under salinity stress [26]. In another study, enhanced salinity tolerance was observed in transgenic tobacco overexpressing both the glyoxalase I and glyoxalase II genes [19]. These plants were also shown to have increased heavy metal tolerance [27]. In this investigation, the overexpression of the glyoxalase II gene in the oilseed crop, $B$. juncea resulted in successful engineering of salinity tolerance. This work might prove to be beneficial in devising future strategies for the recovery of $B$. juncea with enhanced abiotic stress tolerance.

\section{ACKNOWLEDGEMENTS}

This work was supported by a grant from NATP, Government of India. Mukesh Saxena is grateful to the University Grants Commission, India for providing a fellowship. We are also grateful to Prof. Deepak Pental for his initial training in Brassica transformation at CGMCP, Mangolpuri, India. Maintenance of the transgenic plants at the National phytotron facility, IARI, New Delhi and discussions with Prof. Shyam Prakash are gratefully acknowledged.

\section{REFERENCES}

[1] Vinocur B, Altman A. Recent advances in engineering plant tolerance to abiotic stresses: achievements and limitations. Curr Opin Biotechnol 2005; 16: 123-32.

[2] Apse MP, Blumwald EE. Engineering salinity tolerance in plants. Curr Opin Biotechnol 2000; 13: 146-50.

[3] Garg AK, Owens TG, Ranwala AP, Choi YD, Konchian LV, Wu RJ. Trehalose accumulation in rice plants confers high tolerance to different abiotic stresses. Proc Natl Acad Sci USA 2002; 99: 15898-903.
[4] Thornalley PJ. Glyoxalase system new developments towards functional characterization of metabolic pathways fundamental to biological life. Biochem J Kasu 1990; 269: 1-11.

[5] Szent-Gyorgi A, Egyud LG, McLaughlin JA. Keto-aldehyde and cell division. Science 1967; 155: 539-41.

[6] Szent-Gyorgi A. Bioelectronics and cancer. J Bioenerg 1973; 4: 533-62.

[7] Baskaran S, Balasubramanian KA. Effect of methylglyoxal on protein thiol and amino groups in isolated rat erythrocytes and activity of various brush border enzymes. Indian J Bichem Biophys 1990; 27: 13-7.

[8] Kalapos MP. Methylglyoxal toxicity in mammals. Toxicol Lett 1994; 73: 3-24.

[9] Kang Y, Edward LG, Thornalley PT. Effect of methylglyoxal on human leukemia cell growth: modification of G1 growth arrest and induction of apoptosis. Leukemia Res 1996; 20: 397-405.

[10] Milanesa DM, Choudhary MS, Mallouh C, Tazaki H, Konno S. Methylglyoxal induced apoptosis in human prostate carcinoma: potential modality for prostate cancer treatment. Eur Urol 2000; 37: 728-34.

[11] Kalia S, Pal S, Guha-Mukherjee S. Activation of glyoxalase I during the cell division cycle and its homology with auxin regulated genes. Plant Sci 1999; 132: 55-62.

[12] Ramaswamy O, Guha-Mukherjee S, Sopory SK. Correlation of glyoxalase I activity with cell proliferation in Datura callus culture. Plant Cell Rep 1984; 3: 121-4.

[13] Sethi U, Basu A, Guha Mukherjee S. Control of cell proliferation and differentiation by regulating polyamine biosynthesis in cultures of Brassica and its correlation with glyoxalase I activity. Plant Sci 1988; 56: 167-75.

[14] Esparteo J, Sanchez-Aguayo I, Pardo JM. Molecular characterization of glyoxalase I from a higher plant; upregulated by stress. Plant Mol Biol 1995; 29: 1223-33.

[15] Veena, Reddy VS, Sopory SK. Glyoxalase I from Brassica juncea: molecular cloning, regulation and its overexpression confer tolerance in transgenic tobacco under stress. Plant J 1999; 17: 385-95.

[16] Ridderstrom M, Mannervik B. Molecular cloning and characterization of the thioesterase glyoxalase II from Arabidopsis thaliana. Biochem J 1997; 322: 449-54.

[17] Saxena M, Bisht R, Debroy S, Sopory SK, Sarin NB. Cloning and characterization of a mitochondrial glyoxalase II that is upregulated by $\mathrm{NaCl}, \mathrm{Zn}$ and ABA. Biochem Biophys Res Commun 2005; 336: 813-9.

[18] Yadav SK, Singla-Pareek SL, Kumar M, et al. Characterization and functional validation of glyoxalase II from Rice. Protein Exp Purification 2007; 51: 126-32.

[19] Singla-Pareek SL, Reddy MK Sopory SK. Genetic engineering of the glyoxalase pathway in tobacco leads to enhanced salinity tolerance. Proc Natl Acad Sci USA 2003; 100: 14672-7.

[20] Pental D, Pradhan AK, Sodhi YS, Mukhopadhayaya A. Variation amongst Brassica juncea cultivars for regeneration from hypocotyl explants and optimization of conditions for Agrobacterium mediated genetic transformation. Plant Cell Rep 1993; 12: 462-7.

[21] Murray MG, Thompson WF. Rapid isolation of high-molecularweight Plant DNA. Nucleic Acids Res 1980; 8: 4321-5.

[22] Sambrook J, Fritsch EF, Maniatis T. In Molecular Cloning: A Laboratory Manual.(2nd edition) Cold Spring Harbor Laboratory. Cold Spring Harbor, NY 1989.

[23] Chomczinsky P, Sacchi N. Single step method of RNA isolation by acid guanidium thioacyanate-phenol-chloroform extraction. Anal Biochem 1987; 162: 156-9.

[24] Arnon DI. Copper enzymes in isolated chloroplasts: polyphenol oxidase in Beta vulgaris. Plant Physiol 1949; 2: 41-15. 
[25] Yadav SK, Singla-Pareek SL, Ray M, Reddy MK, Sopory SK. Methylglyoxal levels in plants under salinity stress are dependent on glyoxalase I and glutathione. Biochem Biophy Res Commun 2005; 337: 61-7.

[26] Yadav SK, Singla-Pareek SL, Reddy MK, Sopory SK. Transgenic tobacco plants overexpressing glyoxalase enzymes resist an increase in methylglyoxal and maintain higher reduced glutathione levels under salinity stress. FEBS Lett 2005; 579: 6261-5.

[27] Singla-Pareek SL, Yadav SK, Pareek A, Reddy MK, Sopory SK.

Transgenic tobacco overexpressing glyoxalase pathway enzymes grow and set viable seeds in Zinc-spiked soils. Plant Physiol 2006; 140: 613-23.

Received: March 16, 2011

Revised: April 09, 2011

Accepted: April 12, 2011

(c) Saxena et al.; Licensee Bentham Open.

This is an open access article licensed under the terms of the Creative Commons Attribution Non-Commercial License (http://creativecommons.org/licenses/by$\mathrm{nc} / 3.0 /$ ) which permits unrestricted, non-commercial use, distribution and reproduction in any medium, provided the work is properly cited. 